\title{
PMD Effect on the Clock-based Optimum Dispersion Compensation Monitoring Technique
}

\author{
Sung-Man Kim* \\ Department of Electrical and Electronic Engineering, Kyungsung University, \\ Daeyeon 3 dong, Nam-Gu, Busan 608-736, Korea
}

(Received January 29, 2009 : revised February 9, 2009 : accepted February 9, 2009)

\begin{abstract}
We investigate the effect of polarization-mode dispersion (PMD) on the optimum dispersion compensation (ODC) monitoring and nonlinear penalty in optical transmission systems. We report that PMD may reduce the fiber nonlinearity. We also report that the monitoring error of the clock-based ODC monitoring technique decreases after the first-order PMD compensation. A simple explanation of this phenomenon is shown.
\end{abstract}

Keywords: Polarization-mode dispersion (PMD), Fiber nonlinearity, Chromatic dispersion monitoring, Optimum dispersion compensation

OCIS codes : (060.2330) Fiber optics communications; (060.2360) Fiber optics links and subsystems; (060.4370) Nonlinear optics, fibers; (060.4510) Optical communications

\section{INTRODUCTION}

In high-speed optical transmission systems with $\geq 40$ $\mathrm{Gb} / \mathrm{s}$ per channel, it is essential to monitor and compensate for chromatic dispersion in real time $[1,2]$. In the linear optical transmission systems with a low signal power, a 100-\% dispersion compensation gives the best signal quality. Thus, the optimum dispersion compensation is $100 \%$ in the ideal linear optical transmission. However, in the real optical transmission systems with $\geq 40 \mathrm{~Gb} / \mathrm{s}$ per channel, the optical signal power should be high enough to satisfy the required optical signal-tonoise ratio. The high optical signal power causes fiber nonlinearity such as self-phase modulation (SPM), which shifts the optimum value of chromatic dispersion compensation from $100 \%$ to another value [3].

Therefore, finding the optimum value of chromatic dispersion compensation is more important than merely monitoring chromatic dispersion in the real nonlinear optical transmission systems. We have defined the ability to find the optimum value of chromatic dispersion compensation as optimum dispersion compensation (ODC) monitoring [4]. In the earlier works, we have shown that clock-frequency component can be used for ODC monitoring in the nonlinear transmission systems $[4,5]$.

*Corresponding author: sungman@ks.ac.kr
However, we have not considered the polarization-mode dispersion (PMD) effect in the earlier works. Thus we were not able to insist that the clock-based ODC monitoring can be used in the real optical transmission systems with PMD.

In this paper, we investigate the PMD effect on the clock-based ODC monitoring technique. We also investigate the PMD effect on the nonlinear optical transmission. And, a simple explanation about the results are presented.

\section{CONCEPTS AND DEFINITIONS}

In this paper, we introduce several concepts and definitions for a quantitative analysis of chromatic dispersion compensation and fiber nonlinearity, as illustrated in Fig. 1. Dispersion-compensation ratio (DCR) is defined as the ratio of the amount of dispersion compensated by a dispersion compensator to the total amount of dispersion accumulated along the transmission.

In the ideal linear optical transmission systems, a 100-\% DCR gives the lowest eye-opening penalty (EOP). However, in the real nonlinear optical transmission systems, the optimum DCR is shifted from $100 \%$ to another value. The optimum value of DCR is defined as optimum dispersion compensation (ODC) in this paper. And, the tech- 


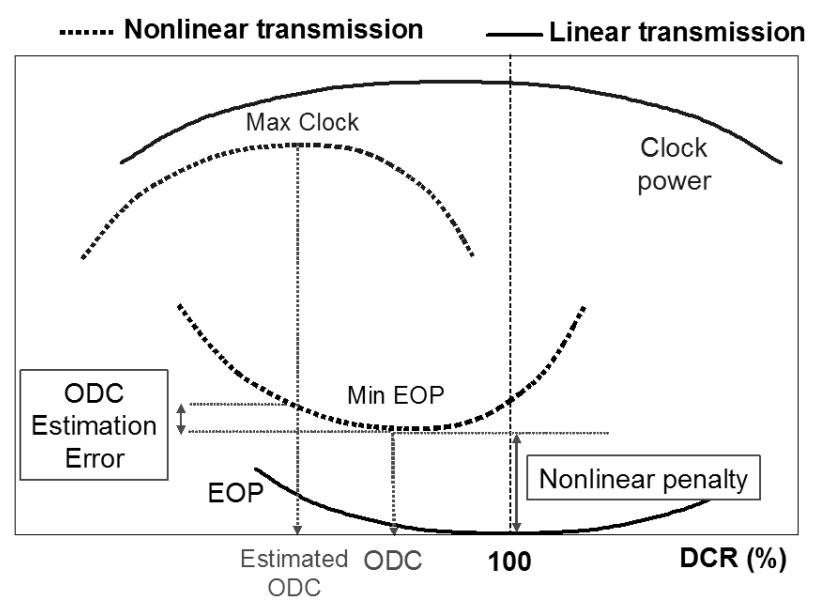

FIG. 1. The definition of nonlinear penalty and $O D C$ estimation error in ODC monitoring. The $\mathrm{X}$-axis is dispersioncompensation ratio (DCR).

nique to monitor the ODC point is defined as $O D C$ monitoring [4].

For a quantitative analysis of fiber nonlinearity, we define the nonlinear penalty as the remained EOP at the ODC point, as illustrated in Fig. 1. The nonlinear penalty implies the minimum EOP that can be obtained by using dynamic chromatic dispersion compensation in nonlinear optical transmission. From the other point of view, it also implies the penalty induced by fiber nonlinearity that cannot be eliminated by chromatic dispersion compensation. We think that the nonlinear penalty can be an index for the amount of fiber nonlinearity.

In the earlier works, we have shown that the ODC point can be detected by finding the peak point of clock power $[4,5]$. In Fig. 1, the estimated $O D C$ means the point detected by finding the peak point of the clock power. However, because the clock-based ODC monitoring technique is not perfect, there exist some errors. To quan- tify the monitoring errors, we define $O D C$ estimation error as the additional EOP induced by the monitoring error of the ODC monitoring technique. The ODC estimation error implies the accuracy of the ODC monitoring technique.

From the next paragraph, we will investigate the PMD effect on the nonlinear penalty and the $O D C$ estimation error in the nonlinear optical transmission systems.

\section{SIMULATION CONDITION}

To investigate the PMD effect, we conducted computer simulations by using a commercial optical transmission simulator, VPItransmissionMaker ${ }^{\mathrm{TM}}$ [6]. We conducted $800-\mathrm{km}$ optical transmission simulations with $40-\mathrm{Gb} / \mathrm{s}$ optical signal. In the simulation, we assume the use of nonzero dispersion-shifted fiber (NZDSF); chromatic dispersion $=4 \mathrm{ps} / \mathrm{nm} / \mathrm{km}$ and PMD $=0.2 \mathrm{ps} /(\mathrm{km})^{0.5}$. The signal format is non return-to-zero (NRZ). The signal power is $-1 \mathrm{dBm}$ and the optical signal is amplified at the end of each 80-km span by an Erbium-doped fiber amplifier. Chromatic dispersion is compensated at the end of the each $80-\mathrm{km}$ span by dispersion-compensating fiber. We assume that PMD is compensated at the end of the total $800-\mathrm{km}$ transmission by the first-order PMD compensator. To investigate the PMD effect statistically, we randomly define 50 sets of the birefringence degrees of fiber to emulate PMD.

\section{RESULT AND DISCUSSION}

Fig. 2 shows a set of the simulation results. One set of the simulation results consists of the EOPs and the clock powers without PMD, with PMD, and with PMD compensation, as a function of dispersion-compensation ratio, in one randomly defined set of the birefringence

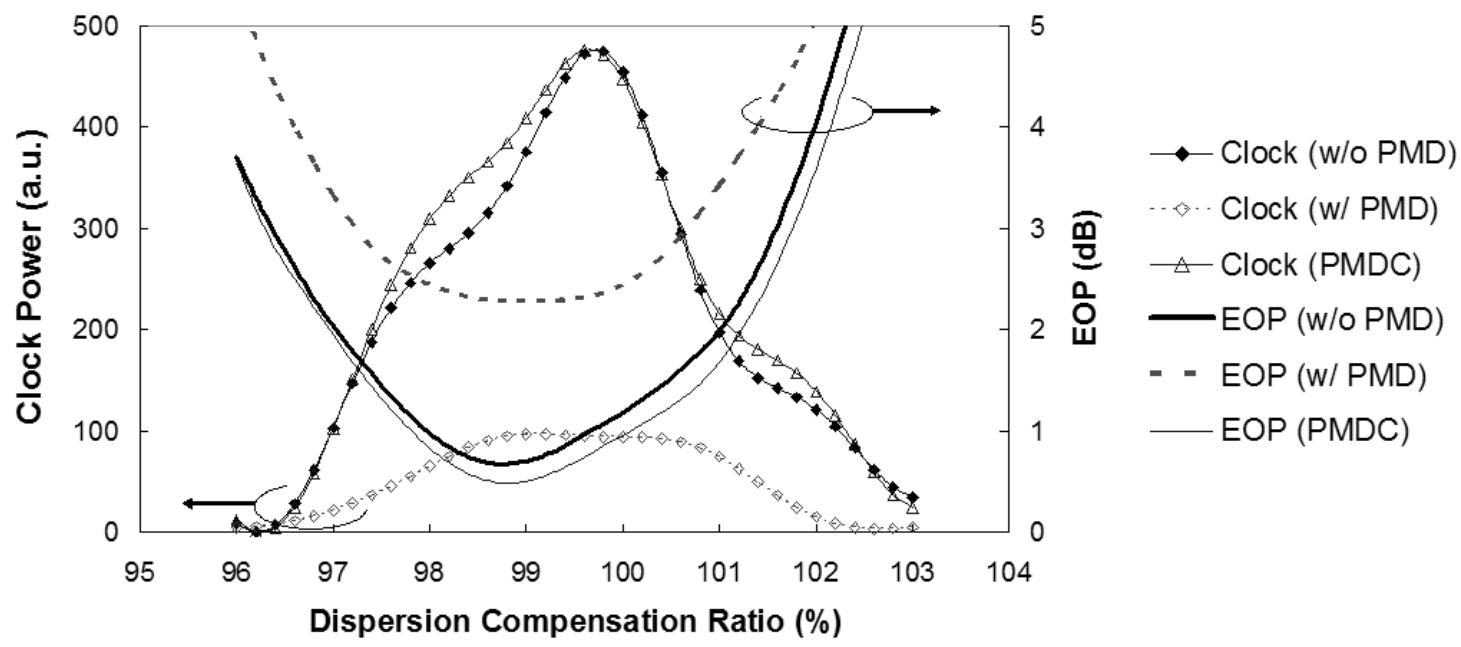

FIG. 2. The EOPs and clock powers as a function of dispersion-compensation ratio without PMD, with PMD, and after PMD compensation (PMDC). 


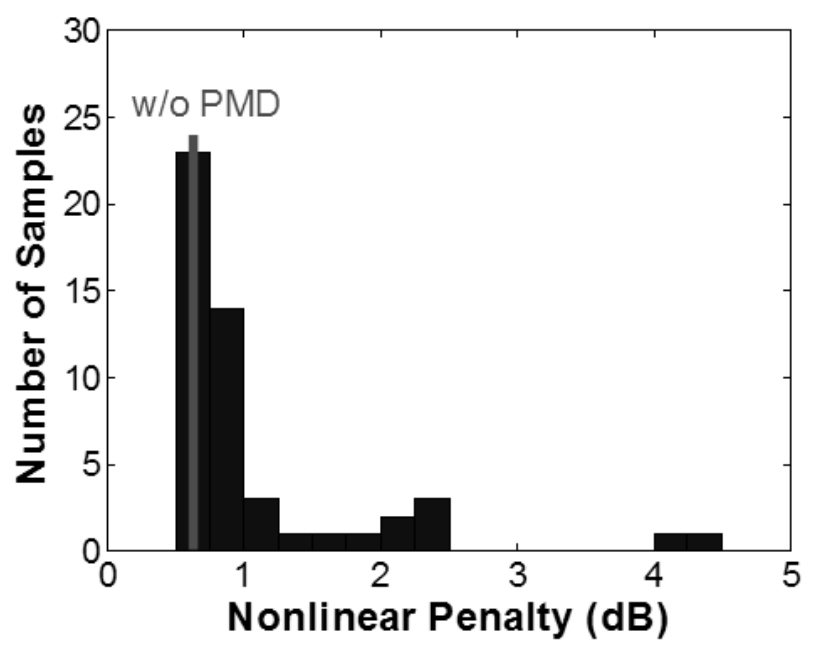

(a) Nonlinear penalties with PMD

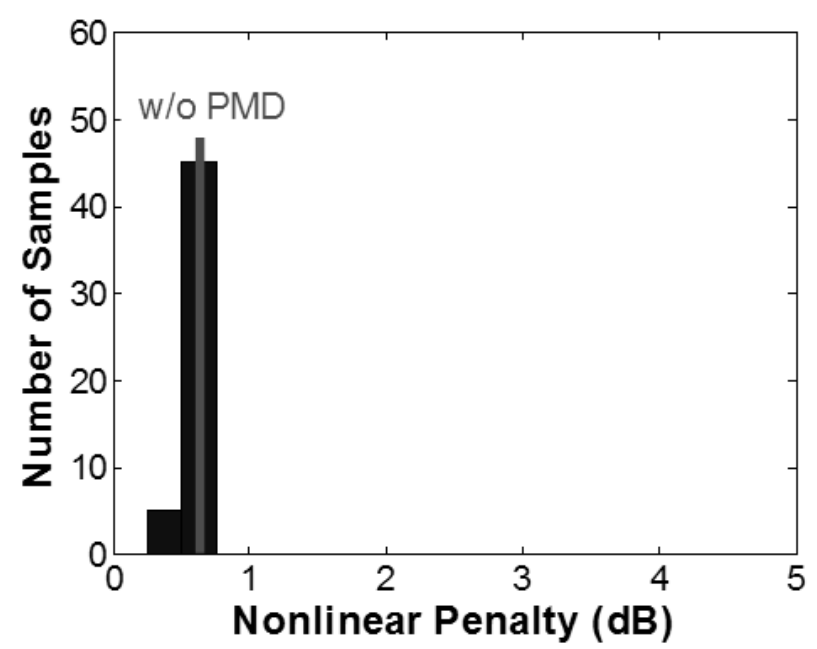

(b) Nonlinear penalties after PMD compensation

FIG. 3. Nonlinear penalties with PMD and PMD compensation. The solid line is the original value without PMD.

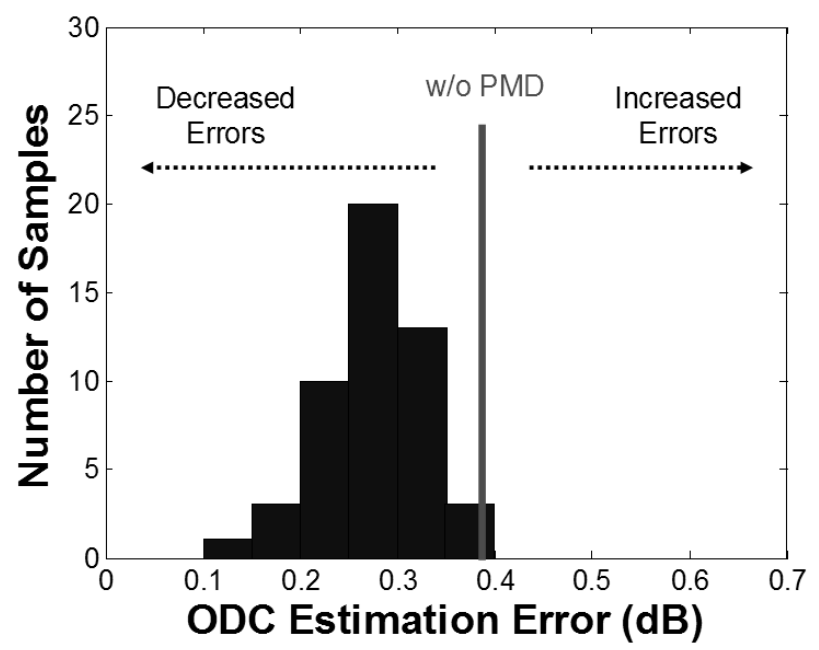

FIG. 4. The ODC estimation errors after PMD compensation degrees of the fiber.

In Fig. 2, the EOPs and the clock powers are displayed when PMD is not modeled, when PMD is modeled, and when the PMD is optimally compensated by a first-order PMD compensator. As shown in Fig. 2, although PMD itself causes severe additional EOP in the optical signal, the EOP after the PMD compensation decreases to even lower than the EOP without PMD. In other words, the nonlinear penalty after the PMD compensation decreases to lower than the original value without PMD. This result shows that PMD may reduce the nonlinear penalty. In Fig. 2, it may be also shown that clock-frequency component could be used to monitor the ODC point after PMD compensation. We will investigate the possibility in Fig. 4.

To investigate the PMD effect statistically, we conducted more simulation sets (total 50 simulation sets) because the result of each PMD simulation can be different due to the birefringence degrees of the fiber although the mean value of PMD is fixed. Fig. 3 shows the nonlinear penalties of the 50 simulation sets with (a) PMD and (b) PMD compensation. It is shown that although PMD itself raises the nonlinear penalties, the nonlinear penalties after the PMD compensation decrease lower than the original value without PMD. It could be reported that although PMD itself is a kind of impairment in optical fiber transmission, PMD has a function of reducing fiber nonlinearity.

Fig. 4 shows the ODC estimation errors after the PMD compensation. It is shown that ODC estimation errors after PMD compensation decrease to lower than the original value without PMD. In other words, clockbased ODC monitoring technique becomes more accurate after PMD compensation, compared with the case without PMD. Thus, it can be reported that the clockbased ODC monitoring technique can be used in the optical transmission systems with PMD compensation.

From the simulation results in Fig. 3 and Fig. 4, it could be thought that PMD may reduce the fiber nonlinearity. To explain this phenomenon, we show a simple explanation in Fig. 5. For an easy explanation, we assume that the optical power is divided between two polarizations ( $\mathrm{X}$ and $\mathrm{Y}$ axis) each with a half power. The nonlinear propagation equations in the fiber with birefringence are given by [7]

$$
\begin{aligned}
& \frac{\partial A_{x}}{\partial z}+\beta_{1} \frac{\partial A_{x}}{\partial t}+\frac{i}{2} \beta_{2} \frac{\partial^{2} A_{x}}{\partial t^{2}}+\frac{\alpha}{2} A_{x}=i \gamma\left(\left|A_{x}\right|^{2}+\frac{2}{3}\left|A_{y}\right|^{2}\right) \cdot A_{x} \\
& \frac{\partial A_{y}}{\partial z}+\beta_{1} \frac{\partial A_{y}}{\partial t}+\frac{i}{2} \beta_{2} \frac{\partial^{2} A_{y}}{\partial t^{2}}+\frac{\alpha}{2} A_{y}=i \gamma\left(\left|A_{y}\right|^{2}+\frac{2}{3}\left|A_{x}\right|^{2}\right) \cdot A_{y}
\end{aligned}
$$

where $A_{x}, A_{y}, \gamma, \alpha, \beta_{1}$ denote the envelope of the TE and TM polarized electrical field components, the Kerr nonlinearity, the fiber attenuation and the group velocity, 


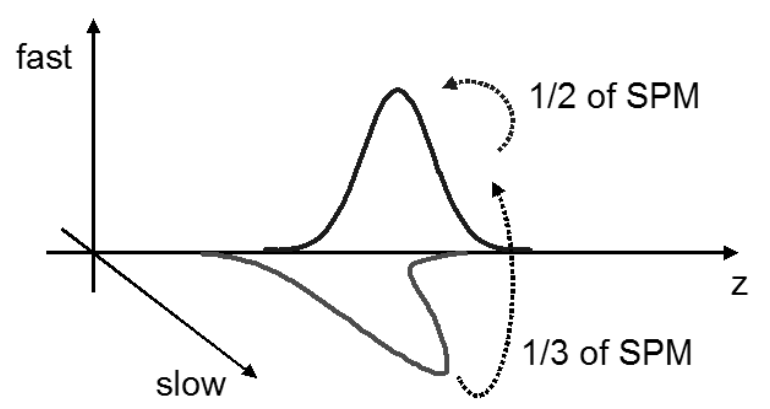

FIG. 5. Nonlinear effect in birefringence of the fiber

respectively, and $\beta_{2}$ is the parameter related to the chromatic dispersion of the fiber.

If we assume that the each polarization has a half power, the total nonlinear effect becomes a $5 / 6(=$ $\left.1^{*} 1 / 2+2 / 3^{*} 1 / 2\right)$ of the original value after the optical signal is divided into two polarizations. In any cases, the total nonlinear effect cannot exceed the original value due to the factor, $2 / 3$ of $\left|\mathrm{A}_{\mathrm{y}}\right|^{2}$. And also, since the pulse positions of the two pulses are different in the Z-axis, the nonlinear effect from the Y-axis pulse cannot affect the whole value to the $\mathrm{X}$-axis pulse. We have found that a similar explanation was reported before [8].

It may be necessary to note that we conducted more simulation sets in other simulation conditions including wavelength-division multiplexing (WDM) conditions, and we confirmed our results.

\section{CONCLUSION}

Using numerical simulations, we investigated the PMD effect on ODC monitoring and fiber nonlinearity. It has been shown that PMD may reduce the fiber nonlinearity. And it also has been shown that the clock-based
ODC monitoring technique become more accurate after PMD compensation. Therefore, we can conclude that clock-based ODC monitoring technique will be useful in the optical transmission systems with PMD compensation. A simple explanation about the phenomenon has been shown.

\section{ACKNOWLEDGMENT}

This research was supported by Kyungsung University Research Grants in 2009.

\section{REFERENCES}

1. C. H. Kim, J. Bae, K. Lee, and S. B. Lee, "Performance evaluation of a tunable dispersion compensator based on strain-chirped fiber Bragg grating in a $40 \mathrm{~Gb} / \mathrm{s}$ transmission link," J. Opt. Soc. Korea 12, 244-248 (2008).

2. K.-S. Kim, J. Lee, W. Chung, and S. Kim, "An electronic domain chromatic dispersion monitoring scheme insensitive to OSNR using kurtosis,” J. Opt. Soc. Korea 12, 249-254 (2008).

3. I. Kaminow and T. Li, Optical Fiber Telecommunications IV B (Academic Press, 2002), Chapter 13.

4. S.-M. Kim and J.-Y. Park, "Chromatic dispersion monitoring of CSRZ signal for optimum compensation using extracted clock-frequency component," ETRI J. 30, 461468 (2008).

5. S.-M. Kim and C.-H. Lee, "The efficient clock-extraction methods of NRZ signal for chromatic dispersion monitoring," IEEE Photon. Technol. Lett. 17, 1100-1102 (2005).

6. http://www.vpisystems.com

7. G. P. Agrawal, Nonlinear Fiber Optics (Academic Press, 2001), Chapter 6.

8. L. Moller, Y. Su, G. Raybon, S. Chandrasekhar, and L. L. Buhlm, "Penalty interference of nonlinear intra-channel effects and PMD in ultra high-speed TDM systems," Electron. Lett. 38, 281-283 (2002). 